\title{
QUANTIFYING BACKGROUND COMPONENTS OF LOW-LEVEL GAS PROPORTIONAL COUNTERS
}

\section{PÁLL THEODÓRSSON}

Science Institute, University of Iceland, Dunhaga 3, 170 IS-Reykjavík, Iceland

\begin{abstract}
I discuss background components of low-level gas proportional counters and show how each component can be estimated based on available data. For more reliable background analysis, further studies are suggested. Based on new information, a generation of low-level gas proportional counting systems for radiocarbon dating may emerge with lower and more predictable background.
\end{abstract}

\section{THE PRESENT STATUS OF GAS PROPORTIONAL COUNTING}

After almost four decades of gas proportional counting and limited new development during the last 10 to 15 years, it seems natural to assume that this technique rests on a solid experimental as well as theoretical basis. What, then, is its present status? An excellent review was given eight years ago by Willem G. Mook (1983), who reported on the performance of most gas proportional counters used for radiocarbon dating.

As working pressure varies from about 1 to $5 \mathrm{~atm}$, the background must be normalized before a comparison of counting systems is made. Mook calculated the expected background at a pressure of $1 \mathrm{~atm}$, the normalized background, $\mathrm{B}_{\mathrm{N}}$. He assumed, based on limited available data, that the background increases by $15 \%$ for each atm increase in working pressure. The effective volume of the counters is found from the count rate of the oxalic acid standard.

I divide the counters into three groups (Fig. 1): 1) ordinary counters in surface laboratories (the largest group); 2) counters in underground laboratories (with more than $10 \mathrm{~m}$ water-equivalent of earth or rock above); and 3) five counters in a surface laboratory in Trondheim with a background record that deserves special attention.

The backgrounds of these three groups of counters is plotted versus their volumes in Figure 1. For counters in surface laboratories, the scatter of background values is shockingly large, considering the great effort presumably invested in each system. Indeed, gas counting was declared "almost obsolete" at the Radiocarbon Conference in Dubrovnik in 1988. However, I believe that gas proportional counters will be important in radiocarbon dating for many years to come because of the potential for lowering background and simplifying the systems.

\section{BACKGROUND COMPONENTS}

The main features of gas-proportional systems are:

1. A central sample counter surrounded by a guard counting system, usually proportional counters, for eliminating the muon component of cosmic rays

2. A layer of old lead or mercury $(2-4 \mathrm{~cm})$ between the guard and sample counter (inner shield)

3. A boronated paraffin neutron-absorbing layer $(5-10 \mathrm{~cm})$

4. Main shield, $10-15 \mathrm{~cm}$ of lead (or $20-30 \mathrm{~cm}$ of iron).

The background, $\mathrm{B}$, of these systems has the following components:

$$
\mathrm{B}=\mathrm{B}_{\beta}+\left\{\mathrm{B}_{\gamma}(\mathrm{E})+\mathrm{B}_{\gamma}(\mathrm{C})+\mathrm{B}_{\gamma}(\mathrm{Rn})+\mathrm{B}_{\gamma}(\mathrm{S})\right\}+\mathrm{B}_{\mathrm{n}}+\mathrm{L}
$$




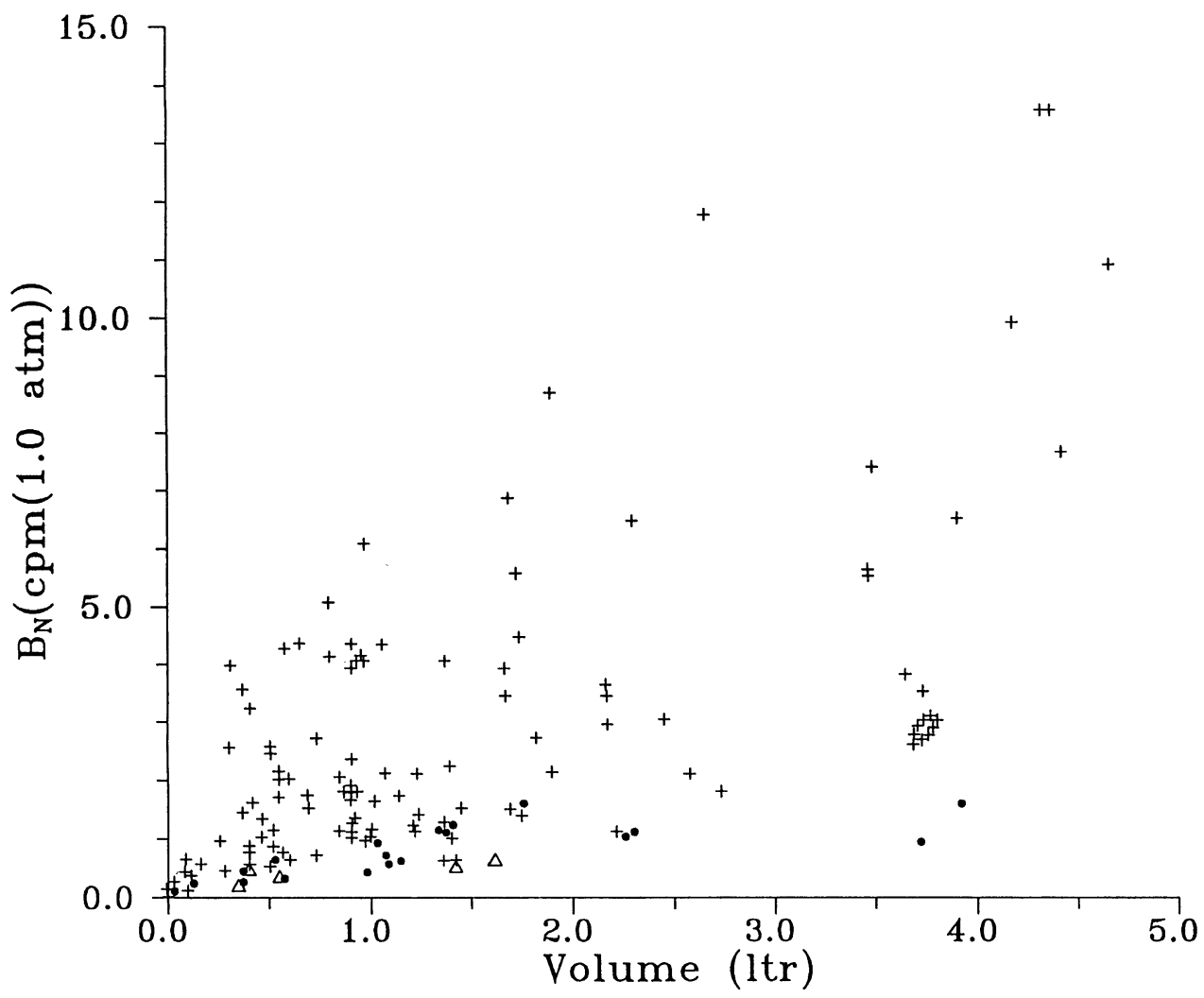

Fig. 1. Comparison of background in gas proportional counters. + - ordinary counters in surface laboratories; - - counters in underground laboratories; $\Delta$ - counters in Trondheim dating laboratory.

where:

$\mathrm{B}_{\beta} \quad=\beta$ activity in the wall of the sample counter

$\mathrm{B}_{\gamma}(\mathrm{E}) \quad=$ radioactive materials outside main shield

$B_{\gamma}(C)=$ contamination by $\gamma$-active nuclides in shield and materials inside it

$\mathrm{B}_{\gamma}(\mathrm{Rn})=$ radon that diffuses into cavities in the shield

$B_{\gamma}(S)=$ secondary $\gamma$ radiation formed by muons and protons in the shield

$\mathrm{B}_{\mathrm{n}} \quad=$ neutrons

$\mathrm{L} \quad=$ muon leakage, i.e., muons that escape detection in guard counter.

A review of the literature has not revealed reliable quantitative determination of any of the background components. On the contrary, the technique is still highly empirical. With modern experimental methods, it is possible to collect much better information about each background component.

\section{BETA CONTAMINATION IN THE COUNTER WALL}

Selection of pure material for the sample counter has rested on common experience of the radioactive purity of different materials. Long considered to have the lowest contamination level of counter materials, quartz has often been used for low-level proportional counters despite its fragile, non-conducting characteristics. Copper is, however, most popular, and steel is frequently used. 
Mook's (1983) comparison shows that counters made of all three materials can give very low backgrounds, but that best results are obtained with copper counters.

Let us look at a typical gas proportional counter with a volume of 1.0 liter and an inner surface area of $770 \mathrm{~cm}^{2}$. A good counter of this size would have a background of $c a .0 .4 \mathrm{cpm}$. If the counter material is contaminated with $60 \mathrm{mBq} \mathrm{kg}^{-1}$ of a $\beta$-emitting radionuclide of $\mathrm{E}_{\max }=1.5 \mathrm{MeV}$, its background contribution would be $c a .0 .04 \mathrm{cpm}$, which can be considered negligible. But what is the level of contamination in counter materials? It is only after the development of ultralow-level counting systems with Ge crystals more than 10 years ago that the radioactive contamination of materials can be checked below the level where it will affect the background of gas proportional counters. Arthur, Reeves and Miley (1988) found no traces of primordial radionuclides in electrolytically refined copper, only traces of cosmogenic activity below $1 \mathrm{mBq}$ $\mathrm{kg}^{-1}$. It is evident that practically no contribution to the background will come from $\beta$-activity in copper of this type.

\section{GAMMA ACTIVITY OUTSIDE THE SHIELD}

The thickness of the main shield is usually chosen so that the background contribution of external gamma activity, $B_{\gamma}(E)$, is reduced to a negligible level. The main shield is usually $10 \mathrm{~cm}$ of lead or $20 \mathrm{~cm}$ of iron. In addition to that, there is usually a $2-4-\mathrm{cm}$-thick layer (inner shield) of lead or mercury between the anticoincidence counter system (guard counter) and the sample detector. The thickness necessary to reduce $B_{\gamma}(E)$ to a negligible level depends, of course, on the concentration of natural radioactive materials in the building, and these may vary considerably between laboratories. The thickness chosen is based on convention rather than quantitative study of the expected value of $B_{\gamma}(E)$, based on surrounding radioactivity and absorption calculations. It is desirable to quantify this and select a thickness that will reduce the external background component below some given value. Background measurements with a Ge spectrometer shielded by varying thickness of lead can give valuable information on optimum thickness.

\section{GAMMA CONTAMINATION OF THE SHIELD}

Lead or iron is used for the main shield, and mercury or lead for the inner shield. I will concentrate on lead, as measurements show that this is the best shielding material.

Older measurements of radioactive contamination in lead are of doubtful value. At present, three reliable methods exist to determine the radioactive contamination in lead: 1) direct $\gamma$ counting with an ultra-low-level Ge crystal, which is the best but most expensive method; 2) direct counting of the $\alpha$ or $\beta$ particles emitting from a lead plate; and 3) chemical separation with subsequent determination of $\beta$ activity from ${ }^{210} \mathrm{Bi}$ or $\alpha$ activity from ${ }^{210} \mathrm{Po}$. Thus, we can now select lead on the basis of direct radiopurity measurements.

Measurements with a Ge crystal have shown that old lead contains no traces of primordial radionuclides (Arthur, Reeves \& Miley 1988; Heusser 1989). These are evidently effectively separated from lead in the refining process, even with techniques used hundreds of years ago. New lead will, however, always contain ${ }^{210} \mathrm{~Pb}$ (half-life $=22$ years) and its progenies, and its concentration will be the same as its ancestor nuclide, ${ }^{226} \mathrm{Ra}$, in ore. This can be quite different from one lead mine to another, lying in the range $20-500 \mathrm{~Bq} \mathrm{~kg}^{-1}$. Lead from the Swedish Boliden mine is frequently selected for shields of low-level systems because of its low ${ }^{210} \mathrm{~Pb}$ activity, $c a .20 \mathrm{~Bq} \mathrm{~kg}^{-1}$.

How much will the ${ }^{210} \mathrm{~Pb}$ contamination affect the background of low-level gas counting systems? The literature does not seem to answer this question, as shown by the fact that it is considered 
sufficient to state that radiopure or old lead has been used. I have measured this with a specially constructed compact low-level system with box-shaped flow counters working in the Geiger region (Theodórsson 1991). The sample counter (inner dimensions $1 \times 5 \times 13 \mathrm{~cm}^{3}$ ) has copper cathodes, and is sandwiched between two slightly larger guard counters. The counters are shielded by $10 \mathrm{~cm}$ of Boliden lead with a total weight of $300 \mathrm{~kg}$. The ${ }^{210} \mathrm{~Pb}$ contribution to the background is mainly from interaction of bremsstrahlung of the energetic $\beta$ particles from its daughter nuclide, ${ }^{210} \mathrm{Bi}$. This radiation will contribute to the background mainly through electrons released from the wall of the counter into the gas and will therefore be proportional to the cathode area. Interaction with the small mass of gas will be negligible.

The background was measured both with lead containing $270 \mathrm{~Bq} \mathrm{~kg}^{-1}$ (background $0.76 \mathrm{cpm}$ ) and with Boliden lead containing $20 \mathrm{~Bq} \mathrm{~kg}^{-1}$ (background $0.55 \mathrm{cpm}$ ). The background contribution of ${ }^{210} \mathrm{Bi}$ can be found from the difference of these two values, ca. $7 \cdot 10^{-5} \mathrm{cpm} \mathrm{cm}^{-2}$ for each $\mathrm{Bq}$ of ${ }^{210} \mathrm{~Pb}$ in a kilo of lead.

This can now be used to calculate the contribution of the ${ }^{210} \mathrm{~Pb}$ contamination to the background of a typical proportional counter with a volume of 1.0 liter with a cathode area of $770 \mathrm{~cm}^{2}$ in a Boliden shield $\left(20 \mathrm{~Bq} \mathrm{~kg}^{-1}\right)$. This would then contribute $c a .0 .05 \mathrm{cpm}$ to the background through interaction of $\gamma$ photons in the wall. If the interaction in gas at 2 atm is included, the total background contribution will be $c a .0 .06 \mathrm{cpm}$. This constitutes $c a$. $10 \%$ contribution to the background of a good proportional counter (Fig. 1). According to this, Boliden lead is acceptable for low-level gas proportional counters. It is, however, desirable to measure this directly and more accurately with a gas proportional counter.

All materials between shield and sample counter should be selected with utmost care, e.g., materials for the guard counter, neutron absorbing layer and construction parts. A system with external guard counters will eliminate these components, as I discuss below.

\section{SECONDARY $\gamma$-RADIATION}

High-energy cosmic-ray muons and protons produce electron-photon showers in the shield. The contribution of these $\gamma$ rays to the background is probably a significant portion of the background of low-level systems.

The significance of the contribution of secondary radiation to the background can be seen from the fact that the background of counters in underground laboratories, where the muon flux has been reduced to $30-90 \%$ of the surface value, is clearly lower than the background of surface counters (Fig. 1).

Measurements using ultra-low-level Ge crystal systems with guard counters on the outer sides of the main shield (Arthur, Reeves \& Miley 1988) provide more direct evidence of the influence of secondary radiation on the background. Muons and protons that produce secondary radiation in the shield are then detected by guard counters as they enter the shield, thus eliminating their contribution to the background. Secondary radiation formed outside the shield is absorbed almost completely by the shield. This arrangement reduces the anticoincidence background of the Ge crystals $90-95 \%$ (Heusser 1989). Scholz (1961) first proposed the use of external guard counters for low-level gas proportional counting systems, but ultra-low-level Ge counters are the first systems constructed with this arrangement. The inner shield of low-level systems with gas proportional counters can, however, be considered a variation of this arrangement, but even 4-5 $\mathrm{cm}$ of lead does not reduce the contribution of the secondary radiation sufficiently. 
It is important to be able to estimate quantitatively the value of the secondary radiation background contribution in gas proportional counters. At low gas pressure and in small counters, this contribution is proportional to the flux of cosmic rays and to the cathode area of the counter, as direct $\gamma$ interaction with the gas is negligible. I have measured this component with the low-level Geiger counter system described above. The anticoincidence background of the sample counter was measured under conditions of varying cosmic-ray flux, under a thin wooden roof, with varying thickness of building materials, and under thick earth and rock layers. Figure 2 shows the anticoincidence background of the sample detector as a function of the coincidence count rate divided by the horizontal cross-section of the sample detector, $\mathrm{cpm} \mathrm{cm}{ }^{-2}$. The contribution of secondary radiation is the only background component that varies and is proportional to the cathode area. If we take point B in Figure 2 as typical for low-level laboratories (with $c a .70 \mathrm{~cm}$ of concrete above), then the background contribution of secondary radiation in the Geiger system is $0.40 \mathrm{cpm}$. As the total cathode area of the sample counter is $166 \mathrm{~cm}^{2}$, this gives $2.4 \cdot 10^{-3} \mathrm{cpm}$ $\mathrm{cm}^{-2}$ of cathode area.

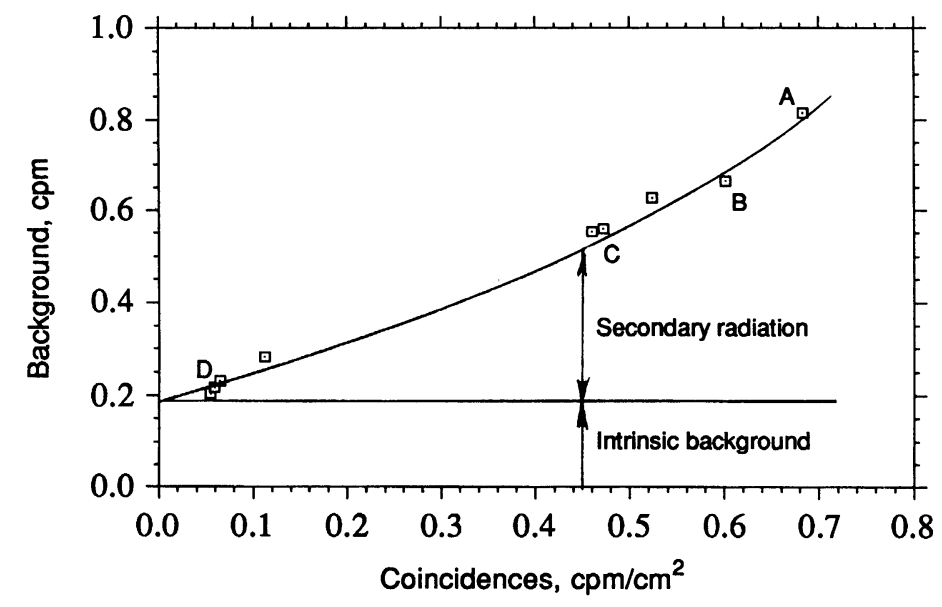

Fig. 2. Background of a low-level Geiger counting system at varying cosmic-ray flux. A. System under a thin roof; B. In basement with 2 stories above; C. In basement with 7 stories above; D. Underground ( $c a$. $100 \mathrm{~m}$ water equivalent)

Using this value, the background of a 1.0-liter gas proportional counter under a similar shield of building materials can be calculated as $c a .2 .0 \mathrm{cpm}$ for the wall component. Direct interaction of $\gamma$ rays with the gas would increase this $c a .20 \%$ at a pressure of $2 \mathrm{~atm}$, leading to a total value of $2.4 \mathrm{cpm}$ from secondary radiation.

\section{NEUTRON COMPONENT}

Neutrons are formed as well as $\gamma$ rays in the interaction of muons and protons in the shield of lowlevel systems, and these will contribute to the background, as shown by de Vries (1956, 1957). Although it is 39 years since de Vries' pioneering work, we still have limited quantitative information about this component. The neutron background contribution can, however, be estimated from de Vries' data and from a Trondheim study (Nydal, Gulliksen \& Lövseth 1977), where the background was measured with both $\mathrm{CO}_{2}$ and propane at the same pressure. The $\gamma$ contribution will be the same for both gases, as the counter will contain an equal mass of gas in both cases, but neutron contribution should be ca. 6 times higher in propane because of a higher cross-section in the hydrogen-rich gas. On the basis of these studies, I have estimated that the neutron component in a surface laboratory under only a thin roof will be $0.4-0.6 \mathrm{cpm} \mathrm{liter}^{-1}$ of $\mathrm{CO}_{2}$ (Theodórsson 1991). 
It seems likely that a major portion of the neutrons near the surface of the Earth are produced by protons, although they are much less abundant than muons. The protons have an attenuating thickness of $120 \mathrm{~g} \mathrm{~cm}^{-2}$, so a good cellar affords considerable neutron protection. This is demonstrated by the very low background of five counters in the Trondheim laboratory which have no neutron absorbing-shield (Fig. 1). Thus, it is questionable if the neutron-absorbing layer is worthwhile in laboratories located in a good cellar, considering that the paraffin layer adds considerably to the total weight of the shield and the complexity of the system.

An external guard counter system is effective in reducing the neutron as well as secondary radiation component of the background (Theodórsson \& Heusser 1991); this component was reduced to $18 \pm 5 \%$ in an ultra-low-level Ge crystal system with external guard counters.

\section{MUON LEAKAGE}

A small percentage of muons crossing the sample counter may not be detected by the guard counting system, causing a muon leakage. Even a small muon leakage may result in a substantial contribution to the background. A good counter with a volume of 1.0 liter will have a background of $c a .1 \mathrm{cpm}$ (Fig. 1) and a total muon count rate of $200 \mathrm{cpm}$. In this case, a $0.1 \%$ muon leakage will increase background by $20 \%$. Can we be sure that the guard counting systems are really that effective?

It is difficult to measure the muon leakage quantitatively. The pulse-height spectrum of the gas proportional sample counter may, however, give an indication of such a leakage, as first reported by Robinson (ms.). The muons give a narrow peak in the coincidence spectrum of the sample counter. A trace of this peak, superimposed on the anticoincidence background spectrum, will reveal even a small muon leakage. More work in this direction is desirable.

A more direct method of measuring muon leakage might be to replace the sample counter with a $7.5 \mathrm{~cm} \times 7.5 \mathrm{~cm} \mathrm{NaI}$ scintillation detector and observe the spectrum from $3-30 \mathrm{MeV}$, as can be seen in work of Stenberg and Olsson (1968), who studied the background of a NaI crystal in a low-level shield. The crystal was shielded with an anticoincidence system consisting of an umbrella of Geiger guard counters. A 5-cm-thick layer of lead was between the NaI crystal and the Geiger counters, in addition to the outer shield of $10 \mathrm{~cm}$ lead. The geometrical shielding of the guard system was well below $100 \%$. In addition to the energy range of radioactive isotopes (below 3 $\mathrm{MeV}$ ), they also measured the pulse-height spectrum up to $40 \mathrm{MeV}$, and recorded both the anticoincidence and total spectrum. Their measurement clearly showed not only the muon leakage (9\%), but also the efficiency of the guard system in reducing the secondary radiation. This is an attractive technique for measuring directly the muon leakage of low-level gas proportional counter system. It may, however, impose practical difficulties.

\section{RADON BACKGROUND COMPONENT}

Without proper precautions, radon can diffuse into the shield of low-level counting systems and raise the background, as shown by Freundlich (1972). The large surface area of gas proportional counters, often at high negative voltage, will collect aerosols with radon progenies that can cause small background fluctuations. In order to minimize this component, the cavities surrounding the sample counter should be made as small as possible. Further, the diffusion of radon into the shield can be obstructed by covering it with a plastic foil. Maintaining good ventilation with a slight overpressure in the counting room will minimize the radon concentration (Freundlich 1972). 
It is desirable to get quantitative information about this component. At what radon concentration in the laboratory air will this component become significant? In order to test this, air might be bubbled at a constant rate through a ${ }^{226} \mathrm{Ra}$ solution into the shield and the increase in background measured, as well as the radon concentration inside the shield. Finally, the radon concentration in the shield should be monitored occasionally during normal work to determine whether precautions should be made to hinder the diffusion of radon into the shield.

\section{INTERLABORATORY COLLABORATION}

Better quantitative knowledge of all background components is now within our reach. New background studies can lead to gas proportional systems with lower and more uniform background. A systematic study should be organized among laboratories with gas proportional counters. Measurements should include: 1 ) recording the anticoincidence as well as the coincidence spectrum of the sample counter to determine eventual muon leakage; 2) pulse-height spectrum of a $\mathrm{NaI}$ crystal where the sample counter has been replaced by the NaI unit, in order to determine eventual muon leakage and partial elimination of the secondary radiation by the anticoincidence arrangement; 3) determining the neutron component by measuring the background with both $\mathrm{CO}_{2}$ and propane; 4) study of radon in the laboratories; and 5) study of the dependence of background with gas pressure, as this is a measure of the $\gamma$-component of the background, independent of the counter volume and its material.

\section{FINAL REMARKS}

In the next few years, I hope we will see a new generation of low-level multidetector systems with gas proportional counters for radiocarbon dating. The counters will be made of electrolyticallyrefined copper (OFHC) that has been tested for radiopurity. Flat-guard gas proportional counters will cover all outer sides of the shield. I would expect the background of a 1.0-liter counter to be about $0.3 \mathrm{cpm}$. The total weight of the shield of a system with eight detectors would be 1200-1500 $\mathrm{kg}$. As the background will be approximately proportional to the cathode area, the background of counters with different volumes will be given by $\mathrm{B}=0.3 \cdot \mathrm{V}^{2 / 3} \mathrm{cpm}$. The higher counting capacity of these systems will increase accuracy and allow more frequent measurements for checking the proper functioning of the systems. I hope that results of development of such systems will be reported at the next Radiocarbon Conference. 


\section{REFERENCES}

Arthur, R. J., Reeves, R. H. and Miley, H. S. 1988 Use of low-background germanium detectors to preselect high-radiopurity materials intended for constructing advanced ultra low-level detectors. IEEE Transactions on Nuclear Science 35(1): 582-585.

de Vries, H. 1956 The contribution of neutrons to the background of counters used for $\mathrm{C}-14$ age measurements. Nuclear Physics 1: 477-479.

de Vries, H. 1957 Further analysis of the neutron component of the background of counters used for C14 age measurements. Nuclear Physics 3: 65-68.

Freundlich, J. C. 1972 Natural radon as a source of low level laboratory contamination. In Rafter, T. A. and Grant-Taylor, T., eds., Proceedings of the 8th International ${ }^{14} \mathrm{C}$ Conference. Wellington, New Zealand, Royal Society of New Zealand: 537-546.

Heusser, G. 1989 The radioecology of natural and artificial radionuclides. In Feldt, W., ed., Proceedings of the XV Regional Congress of IRPA. Fachverband für Strahlenschutze V: 468-463.

Mook, W. G. 1983 International comparison of proportional gas counters for ${ }^{14} \mathrm{C}$ activity measurements. In Stuiver, M. and Kra, R. S., eds., Proceedings of the 10th International ${ }^{14} \mathrm{C}$ Conference. Radiocarbon
25(2): 475-484.

Nydal, R. 1962 Review of Scientific Instruments 33: 1313.

Nydal, R., Gulliksen, S. and Lövseth, K. 1977 Proportional counters and shielding for low level gas counting. In Povinec, P. and Usacev, S., eds., Proceedings of the International Conference on Low-Radioactivity Measurements and Applications. High Tatras 1975. SPN, Bratislava: 77-84.

Robinson, S. W. (ms.) Pulse-height analysis for gas proportional counters. Abstract. Radiocarbon 33(2): 237.

Scholz, T. 1961 Untersuchung des Nulleffektes in Szintillationszählern un Zählrohren, Diplomarbeit, Heidelberg, II. Physikalisches Institut.

Stenberg, A. and Olsson, I. U. 1968 A low level gamma counting apparatus. Nuclear Instruments and Methods 61: 125-133.

Theodórsson, P. 1991 The background of gas proportional counters. Journal of Physics 17: 419-427.

Theodórsson, P. and Heusser, G. 1991 External guard counters for low-level counting systems. Nuclear Instruments and Methods B53: 97-100. 